E.L.U.A., 1, 1983, págs. 303-324

\title{
SOBRE EL TRATAMIENTO DE LA SINTAXIS EN ALGUNAS PROPUESTAS GENERATIVAS
}

\author{
Agustín Vera Luján \\ (Universidad de Murcia)
}

0. En la historia reciente de la Lingüística, el desarrollo de las llamadas Gramáticas Generativas Transformacionales (GGT) ha supuesto un, si no absoluto, sí considerable progreso en la preocupación por el rigor formal de las formulaciones gramaticales; un rigor inmediatamente exigido por la naturaleza deductiva de los presupuestos metodológicos últimos de la GGT, y que habría de tener como otra de sus consecuencias innegablemente positivas un ensanchamiento paralelo en la amplitud de miras de las preocupaciones de la Lingüistica, nunca, ciertamente, ajena a las conexiones de los procesos gramaticales con otros más generales como los psicológicos o psicolingüísticos, los filosóficos, epistemológicos, etc., pero en pocas ocasiones, sobre todo por lo que se refiere a la historia lingüística más reciente, más cómoda y convencidamente instalada en la convicción de la necesidad de un planteamiento interdisciplinario de su campo de trabajo.

0.1. Afirmar que la vertiginosa evolución teórica de la GGT no ha supuesto, en un saldo final, modificaciones sustanciales importantes - con la excepción de algunas de las precisiones aportadas por la denominada "Semántica Generativa" - para la imagen última de la GGT tal y como, básicamente, fuera esbozada por Noam Chomsky en 
Aspectos de la teoria de la sintaxis podría parecer, tal vez, un contrasentido difícilmente sostenible ante la hoy ya inabarcable cantidad de trabajos en los que las tesis chomskyanas han sido discutidas, matizadas y reformuladas desde el interior del mismo modelo, para conseguir dotarlo de una coherencia y rigor de funcionamiento finalmente incuestionables. Sin embargo, una aproximación crítica a estos hechos no puede por menos que constatar cómo, con la excepción mencionada de algunas de las propuestas de la Semántica Generativa y, de manera más precisa, de la denominada "Gramática de Casos", las precisiones aportadas al modelo chomskyano no han logrado en modo alguno poner al descubierto algunas de sus insuficiencias más graves; contribuyendo, al contrario, al centrarse preferentemente en problemas relacionados con el ajuste de funcionamiento de aspectos particulares dentro del mosaico general del modelo, al inevitable proceso de esclerotización que la búsqueda interna de coherencia conlleva cuando se realiza con unos límites absolutamente prefijados, como en este caso.

0.2. Mi intención en este trabajo es mostrar cómo una perspectiva de mayor alcance crítico para con la meta-teoría de la GGT es necesaria para lograr, no sólo soluciones más satisfactorias a concretos problemas gramaticales cuyo tratamiento en la GGT se traduce en caracterizaciones dudosas, y mantenibles, única y exclusivamente, en evitación de la necesidad de alteración de muchos de los presupuestos fundamentales del modelo chomskyano, en caso de proceder de manera diferente; sino también el acercamiento de los presupuestos sobre los que la GGT descansa explicitamente a aquéllos que la moderna psicologia ha puesto de manifiesto y que, como veremos, chocan frontalmente en ocasiones con los de la GGT; produciéndose además la paradoja de que, desde el punto de vista de una correcta adecuación respecto de los principios básicos de la reciente Psicología cognitiva, tiene lugar una coincidencia con determinadas prácticas o procedimientos estructuralistas que, no sólo contradice la injustificada sanción negativa chomskyana sobre esta metodología lingüistica, sino que, al contrario, apunta, en un sentido diferente, a una inadecuación real por parte de la GGT.

1. Como es sobradamente conocido, la GGT se constituye básicamente como modelo que ha de reflejar la competencia de unos hablan- 
tes que son capaces, a partir de un conocimiento efectivo previo de enunciados de una lengua necesariamente limitado, de producir un número prácticamente ilimitado de enunciados gramaticales de dicha lengua. Semejante capacidad para la comunicación a través de lenguas naturales se supone innata, y, como segunda hipótesis, modelizable en términos de un conjunto limitado de reglas capaces de producir un número virtualmente ilimitado de enunciados, en tanto que reflejo de dicha competencia, como concepto diferente, y separable del de actuación.

1.1. Siendo otro postulado básico en la GGT el de la capacidad para "manejar" una lengua se fundamenta en la capacidad de sus hablantes para formar las oraciones gramaticales de esa lengua, la GGT instituye a la Sintaxis en el componente básico del modelo, confiando el tratamiento generativo de los fenómenos de naturaleza sintáctica a dos tipos de reglas, básicas y transformacionales, cuya operatividad tendría como consecuencia la generación del conjunto virtual de oraciones gramaticales de una lengua.

1.2. En principio, un modelo constituido en esta forma (integrado, en su versión "sintactista" chomskyana, por otros sub-componentes interpretativos como el semántico y el fono-fonológico, y sometido a buen número de restricciones de funcionamiento; aspectos éstos en los que no parece necesario el detenerse por ser sobradamente conocidos) es perfectamente capaz de explicar cuestiones de naturaleza sintáctica en relación con gran número de enunciados; $y$, así, utilizando un par de ejemplos que permitan poner esquemáticamente de manifiesto los aspectos de la operatividad de semejante modelo que puntualmente interesan, la interacción de las reglas del componente sintáctico básico y las reglas transformacionales permitiría, en efecto, respecto de enunciados como (1) y (2), poner de manifiesto cómo:

i) tanto en el caso de (1) como en el de (2), los enunciados estarian estructuralmente organizados mediante la articulación de dos cláusulas distintas;

ii) la estructura sintáctico-funcional de (1) y (2) es semejante; es decir, en ambos casos una de las dos cláusulas depende, o «está dominada", por la otra, respecto de la cual funciona como "objeto directo»; 
y en ambos, también, cada una de las dos cláusulas presenta una estructura del tipo «Sujeto-Predicado";

iii) la especificidad de (1) frente a (2) vendría del hecho de que, siendo en (1) el sujeto de la cláusula dominante idéntico al de la dominada, el de esta última se ve impedido de aparecer en la manifestación lineal del enunciado; lo que conlleva, a su vez, la realización como infinitivo de la forma verbal que desempeña la función de núcleo del Predicado.

(1) Juan quiere salir

(2) Juan quiere que Andrés salga

1.2.1. Los concretos procedimientos utilizados por la GGT para poner de manifiesto estas relaciones son sobradamente conocidos. En sintesis, (1) y (2) tendrian en común una misma representación estructural «profunda», cuya paráfrasis estaría más cerca de (2) que de (1). La aplicación posterior a esa estructura profunda de, entre otras, una transformación actuada por la condición de identidad del sujeto de la cláusula dominada con el sujeto (objeto directo o indirecto, en otras ocasiones) de la dominante tendría como efecto la conversión de tal estructura oracional en otra caracterizada, desde el punto de vista de su manifestación lineal-discursiva, por los rasgos indicados en iii), correspondiente a (1).

2. En términos generales, la validez de un modelo elaborado en esta forma puede considerarse garantizada desde el punto de vista de la corrección de las conclusiones obtenibles, siempre que se mantenga en todo momento, como ya desde un temprano momento en el desarrollo de la GGT se indicara, para los elementos oracionales relacionados mediante el recurso a las transformaciones, la identidad de significado entre la cadena oracional básica y la transformada (1), lo que implicará, necesariamente, la existencia en dichas cadenas o estructuras oracionales básicas de todas las informaciones necesarias para la decisión en el sentido de la posibilidad/necesidad de aplicación de una determinada regla transformacional.

(1) Cf. J. J. KATZ y P. M. POSTAL, An Integrated Theory of Linguistic Descriptions, Cambridge, M.I.T. Press, 1964, pág. 71 y ss. 
2.1. Con tales restricciones básicas desde el punto de vista general que actualmente nos interesa, el modelo generativo-transformacional esbozado por Chomsky permite, de hecho, tratar gran número de problemas sintáctico-gramaticales; específicamente, la generación o explicitación de las relaciones entre todos aquellos enunciados oracionales en los que se mantiene una misma estructura sintáctica subyacente a las operaciones posibles de transformación: básicamente, adición, supresión o cambio de lugar de determinados elementos. Si el universo lingüísticoobjetual a sí misma, asignado por la GGT coincidiera exactamente con tales límites, no existirian mayores problemas, pero desde sus mismos inicios como metodologia, la GGT, junto al tratamiento de problemas escrupulosamente ajustados a tales restricciones, ha dado cabida entre sus supuestas competencias explicativas a la descripción de otros que, aunque tratados por ella como absolutamente semejantes a los precedentes, escapan incuestionablemente a los límites auto-asignados, y son objeto de caracterizaciones problemáticas, tanto desde el punto de vista de su formalización, como porque hacen dudar de la solidez de un modelo capaz de pasar por alto la heterogeneidad de tales cuestiones sin advertirla.

2.2. Como caso concreto de fenómeno cuya consideración estaria fuera de los limites autoconcedidos por la GGT (tomando siempre como marco de referencia la versión estándar chomskyana), tal vez ninguno es más claro que el tipo de construcciones denominadas pasivas, cuya generación constituyó, incluso, uno de los argumentos fundamentales esgrimidos por Noam Chomsky en 1957 para la justificación de la necesidad de un recurso meta-teórico como el de las transformaciones, y que ha sido objeto de numerosísimos estudios posteriores (2).

2.3. Resumiendo brevemente los aspectos fundamentales del problema, la relación entre oraciones activas y pasivas es resuelta en la GGT en los siguientes términos:

(2) Sobre la complejidad de los procesos pasivos y su resistencia a la formalización lingüística, Cf., R. LAKOFF, «Passive Resistence», en Chicego Linguistic Society, 7, 1971, págs. 149-169. 
i) dada una estructura oracional del tipo «SN Aux V SN"; es decir, una estructura oracional de predicado transitivo, y en la que el núcleo del predicado es compatible con adverbios de modo, puede sometérsela a una transformación denominada "Pasiva», que la convertirá en otra cadena oracional donde el SN en función de Objeto Directo en la cadena pre-transformada desempeñará la función de Sujeto gramatical en la cadena transformada, y el Sujeto de la cadena pretransformada la de Sintagma preposicional, o Circunstancial, en la transformada. En términos chomskyanos (3), toda cadena de la forma de (3a) tendría, como resultado de la aplicación de esta transformación, la forma de la $(3 b)$ :

(3)

a) SN-Aux-V-SN-... Por Pasiva

$\begin{array}{lllll}1 & 2 & 3 & 4 & 5\end{array}$

b) $4-2-3-$ por 1

ii) Paralelamente, habrian de ser establecidas algunas restricciones para ajustar el funcionamiento de semejante transformación: en lenguas como el inglés, donde es posible la pasivización de estructuras oracionales asignando la condición de sujeto de la oración pasiva al Objeto Indirecto de la correspondiente cadena activa, la transformación pasiva deberia ser ordenada con relación a la que se ha denominado "Transformación de cambio de lugar del Objeto Indirecto". (4), de manera que pudiera operar sólo tras esta última, puesto que, como se desprende del funcionamiento de (3), el elemento elegido para la función de sujeto de la cadena pasiva es siempre el SN que sigue al verbo ( $V$ ) de la activa; siendo, en consecuencia, necesario que, en el caso mencionado, el SN objeto indirecto de la cadena oracional activa ocupe esa posición para que pueda ser tomado en consideración por la transformación pasiva.

iii) De manera semejante, por último, sería también necesario bloquear la posibilidad de producción de un enunciado como (4) "Si mismo fue destruido por ese hombre, resultado de la aplicación de la transformación pasiva a un enunciado como (5) Ese hombre se destruyó a si mismo; impidiendo que dicha transformación fuese aplicable a estructuras oracio-

(3) Cf. N. CHOMSKY, Aspectos de la teoria de la sintaxis, Madrid, Aguilar, 1970, pág. 101.

(4) Cf. CHARLES J. FILLMORE, Indirect Object Constructions and the Ordering of Transformations, Londres, Mouton, 1965, pág. 14.

(5) Para este tipo de fenómenos, Cf., P. POSTAL, Cross-Over Phenomena, Nueva York, Holt, Rinehart y Winston, 1971. 
nales donde el sujeto y el objeto directo -en el caso de nuestra lengua- fuesen referencialmente idénticos, como es el caso de (5) (5).

3. El tratamiento transformacional de las denominadas "oraciones pasivas" habría contado incluso con una supuesta apoyatura desde el campo de las investigaciones psicolingüísticas. Partiendo de la hipótesis de que toda regla gramatical ocuparía un determinado "espacio de almacenamiento" en la memoria humana, se habría comprobado a través de diversos experimentos cómo el procesamiento de cadenas oracionales en cuya estructura habria de jugar un papel distintivo el fenómeno de la pasividad requeriría una mayor cantidad de tiempo, estaría sujeto a una mayor probabilidad de error, etc., etc., que el de oraciones para las que no es preciso suponer el mismo recurso transformacional (6); de lo que, por lo tanto, habría de deducirse una especie de definitiva validación empirica de la hipótesis de la existencia de un proceso transformatorio entre los operativos en el caso de la estructura oracional pasiva.

4. La descripción del fenómeno de la pasividad en la GGT en los términos a que acabamos de hacer referencia choca, a pesar del considerablemente ajustado tratamiento de este fenómeno en semejante modelo, y de su supuesta validación psicolingüistica, con varios inconvenientes. No entraré en el ya señalado por Lakoff (7) en el sentido de que, en contra de la formulación chomskyana de que sólo son susceptibles de utilización en construcciones pasivas los verbos compatibles con adverbios de modo, buen número de verbos no libremente combinables con este tipo de adverbios son, a pesar de todo, utilizables en construcciones pasivas, toda vez que es posible, incluso, dar la razón a Chomsky sobre la desproporción entre inconvenientes tales y la necesidad de reelaboración total del modelo general para acogerlos o subsanarlos (8). Cabría, en este sentido, pues, soportar esta inconsistencia meta-teórica a la espera, y en la esperanza, de su posible corrección;

(6) Cf. en este sentido J. Mehler, "Some effects of grammatical transformation on the recall of English sentences", en J. verb. Learn. verb. Behav., 2, 1963, págs. 346-351.

(7) Cf. G. LAKOFF, Irreguiarity in syntax, Nueva York, Holt, Rinehart y Winston, 1970, pág. 156.

(8) Sobre el parecer de Noam Chosmky en este sentido, Cf. «Une conception transformationelle de la syntaxe», en Langages, 4, 1966, págs. 78-79. 
pero no todas las resistencias suscitadas a la GGT por las construcciones pasivas pueden ser, desgraciadamente, saldadas con este mismo talante conciliador, ni afectan a cuestiones tan de detalle.

4.1. Especialmente improcedente parece la actitud meta-teórica de la GGT a propósito de la formalización de las relaciones existentes entre oraciones activas y pasivas por lo que supone de contravención de los propios postulados, al relacionar mediante una regla transformacional dos estructuras oracionales con significados siempre diferentes desde el punto de vista oracional: un esquema oracional, el activo, en el que se presenta, en términos greimasianos, la clara atribución a un actante agente de un predicado dinámico, o funcional; frente al esquema oracional pasivo, que admite, al menos, una interpretación en términos de una predicación estática, o cualificativa (9), sin que existan en lugar alguno en el esquema teórico de la GGT mecanismos adecuados para la explicación de semejantes relaciones.

4.2. Ciertamente, la diferente significación de estructuras oracionales activas y pasivas había sido tempranamente advertida (aunque desde una perspectiva básicamente anclada en los aspectos léxicos) (10); y resultado de ello sería la primera gran modificación de las tesis chomskyanas tal y como quedaría reflejada en Aspectos de la teoría de la sintaxis, donde, en evitación de tales problemas, la condición pasiva de una oración no era ya, como en Estructuras sintácticas, un simple añadido producto de la correspondiente transformación, sino que resultaba explicada desde la base misma de la gramática a través de la introducción de los elementos «Por Pasiva», que conllevarian necesariamente la aplicación de la regla transformatoria.

4.3. Sin embargo, un recurso como éste no deja de ser una solución notacional, posiblemente válida en cuanto tal, pero que no supone la eliminación total de la objeción que apuntábamos, puesto que, aún con

(9) Cf. A. J. GREIMAS, Semántica estructural, Madrid, Gredos, 1973, pág. 237.

(10) Cf. J. J. KATZ y P. M. POSTAL, An Integrated..., cit., págs. 72-74. 
la presencia de semejante elemento como supuesto responsable de la introducción de la noción de pasividad en las estructuras oracionales básicas, dichas estructuras son, paradójicamente, formuladas en lo que podríamos considerar como su forma activa, en la que se incluye el constituyente «Por Pasiva» como un añadido, consecuencia de lo cual serian las operaciones de «cambio de lugar» de los distintos constituyentes activos afectados por la transformación.

4.3.1. Así pues, en el marco de la teoría de Aspectos..., al que básicamente nos venimos refiriendo, la caracterización chomskyana de la pasividad se reduce, de alguna manera, en el nivel subyacente, básico, a la indicación contradictoria de que la estructura de tales oraciones es analizable en dos rasgos fundamentales: Presencia del rasgo de pasividad, pero añadido a una estructura oracional de carácter activo; caracterización a todas luces poco o nada satisfactoria para reflejar las conexiones entre dos estructuras sintácticas supuestamente en relación de paráfrasis; lo que implicaria el que ambos esquemas oracionales constituyen dos alternativas igualmente posibles para la expresión de contenidos semejantes; todo lo cual no parece ser adecuadamente reflejado por un tratamiento gramatical según el cual, en el proceso de producción de una oración pasiva, el hablante debe contar de alguna forma con una estructura activa como componente básico de dicho proceso; lo que, en la práctica, equivale a conceder a la estructura activa una preponderancia absoluta que relegaria a la pasiva a la condición de variante, recordando, por tanto, actitudes como las encontrables a propósito de las definiciones o categorizaciones de las formas casuales en las gramáticas clásicas, donde se suponía la existencia de formas no casuales, especie de trasunto lingüístico de los conceptos lógicos invariables - las formas de nominativo-, frente al resto de variaciones propiamente casuales.

5. Lo que sucede es que, a pesar de la propia auto-presentación como metodología superadora de estadios de reflexión lingüistica supuestamente menos satisfactorios (especialmente en relación con la metodología estructuralista), la GGT comenzó a desarrollarse como modelo combinando aspectos de incuestionable novedad - sobre todo, la insistencia en el carácter deductivo de sus planteamientos teóricosjunto con otros, paradójicamente centrales para la construcción del modelo deseado, inmediata y acríticamente asumidos de entre el reper- 
torio categorial de las gramáticas tradicionales, de las que la GGT incorporaría sin mayores distingos(11), de una parte, su repertorio de categorías gramaticales: los elementos primitivos gracias a los cuales son reflejadas las diferentes relaciones sintácticas; de otra, la misma tradicional conceptuación de las distintas funciones sintácticas, que en lo que podríamos calificar de versión "ortodoxa" de la GGT reciben una definición basada simplemente en términos de “dominio» que en nada modifica o supera la doctrina tradicional al respecto, aun a pesar de que sea lícito imaginar un comportamiento distinto, mucho más minucioso y preocupado por la precisa caracterización de los conceptos recubiertos, y no siempre con fortuna, por las distintas etiquetas funcionales tradicionales, en un modelo que asigna a la sintaxis el papel central en el juego de componentes cuya articulación debe hacer posible la generación de todas las oraciones gramaticales de una lengua.

5.1. En cierta forma, el conjunto de aspectos objetables que venimos reseñando en relación con la imagen del funcionamiento sintáctico de una lengua obtenible de la GGT podría ser puesto globalmente en relación con la pervivencia en esta metodología de una concepción de la Sintaxis que, salvadas las diferencias evidentes, está también presente de manera implícita en las aproximaciones tradicionales a los problemas del nivel sintáctico, repetidamente caracterizado como nivel donde simplemente tendria lugar la combinatoria de las unidades de un nivel “inferior", el morfológico; merced a lo cual la tarea de definición de las unidades específicas del componente sintáctico — por lo demás ineludible si se desea su correcta caracterización como tal- puede ser obviada.

5.2. Si el concepto de Sintaxis subyacente a la GGT hubiese estado libre de esa fuerte contaminación morfológica, y el componente sintáctico hubiese sido considerado básicamente desde una perspectiva interesada en el descubrimiento de las especificidades de semejante nivel, hubiera sido claro que si una oración es un signo es porque a una

(11) Hablo, claro está, de los inicios chomskyanos de la GGT; no de las modificaciones y desarrollos posteriores, de los que puede ser un buen ejemplo el ya citado Irregularity.. 
forma determinada corresponde un significado oracional particular; que la consideración de dos esquemas oracionales como sujetos a una relación de paráfrasis supone necesariamente la persistencia de una misma significación oracional, más allá de las diferencias formales existentes entre ellos; y que, por lo tanto, en conclusión, los esquemas oracionales denominados "activos" $y$ "pasivos" no pueden ser confrontados sobre la base de su supuesta condición parafrástica mutua puesto que sus respectivos significados oracionales son, como hemos visto, diferentes.

5.3. La relación entre oraciones activas y pasivas que la versión estándar chomskyana de la GGT ha venido tratando inmutablemente sobre la base de su semejanza de significados, es, como algunas de las más inteligentes apreciaciones críticas de la GGT han puesto de manifiesto (12), una relación que se establece sobre el plano, no del significado oracional, sino de la designación operada por ambos esquemas oracionales respecto de un mismo tipo de referentes sintáctico-funcionales. Por esta razón, uno de los desarrollos teóricos más importantes en el ámbito de la GGT -y que debe ser destacado por su importancia de entre el resto de formulaciones genéricamente reconocidas bajo la etiqueta de "Semántica Generativa» que, al menos hasta hace algunos años (13), se han desarrollado como variantes notacionales de la GGT de inspiración chomskyana - es el debido a Charles J. Fillmore y su modelo de «Gramática de Casos", que, instaurando en el nivel de estructura subyacente oracional un conjunto de relaciones casual-predicativas, constituye el intento de mayor eco y difusión de formalización de un nivel sintáctico-designativo imprescindible en un momento de evolución de la GGT en el que, no limitándose ésta al estudio de relaciones de significado tan sólo, se procedería erróneamente a la descripción de determinadas relaciones entre enunciados - como es el caso de la que nos venimos ocupando- pretendiendo que se operaba siempre dentro de un único nivel explícitamente delimitado por la exigencia de identidad de significado.

(12) Cf., especialmente, E. COSERIU, “Significado y designación a la luz de la semántica estructural", Madrid, Gredos, 1977, págs. 185-209; también CI. HAGEGE, La gramática generativa. Reflexiones criticas, Madrid, Gredos, 1981, pág. 135.

(13) Excluimos, pues, algunos de los desarrollos más recientes de la SG o, al menos, de los trabajos de los, en el pasado, «semantistas generativos». Cf., por ejemplo, G. LAKOFF, "Linguistic Gestalts», en Chicago Linguistic Society, 13, 1977. 
5.4. En el caso concreto de la formalización de las conexiones entre esquemas activos y pasivos, un modelo semejante significaría finalmente la posibilidad de dar cuenta de tales conexiones de forma considerablemente más coherente a como era posible hacerlo dentro de la GGT chomskyana; estableciendo que dos enunciados como (6) y (7) serian realizaciones diferentes de una misma estructura casualpredicativa como (8) sobre la que, en el caso de (6), una transformación de selección de sujeto habría operado sobre el «Agente», y, en el de (7), sobre el "Objetivo", según una serie de instrucciones que determinarían las circunstancias en las que tales transformaciones operarian y que, de acuerdo con Fillmore, establecen que:

i) si existe un Agente, se convierte en sujeto; de otra forma, si existe un Instrumental, es él el que se convierte en sujeto; en otro caso, el sujeto es el Objetivo (14).

ii) Frente a i), que establece las condiciones para la elección normal de sujeto, es posible otra elección diferente, «no-normal», que (para el caso de lenguas como la nuestra) permite la elección del elemento Objetivo como sujeto cuando el verbo es marcado por el rasgo [+ pasiva] (15).

(6) Juan entregó los libros a mi hermano

(7) Los libros fueron entregados a mi hermano por Juan

(8) Verbo, Agente, Objetivo, Dativo

6. La propuesta de Fillmore, con la instauración en el nivel subyacente del componente sintáctico de un conjunto de relaciones casuales capaces de reflejar los aspectos sintáctico-designativos, supone, respecto de la, de otro modo, problemática inclusión de cuestiones heterogéneas bajo una sola perspectiva teórica incapaz de lidiar satisfactoriamente con ellas, la incuestionable ventaja de permitir diferenciar con claridad entre, de un lado, relaciones entre enunciados que, además de poder ser representados como compartiendo una descripción semejante en un nivel sintáctico-designativo, tienen en común su condición de signos sintácticos sinónimos (caso de (1) y (2), donde no sólo se da

(14) Cf. CHARLES J. FILLMORE, "The case for case", en Universals in Linguistic Theory, ed. por E. Bach y R. T. Harms, Nueva York, Holt, Rinehart y Winston, 1968, pág. 33.

(15) Ibíd., pág. 62. 
una identidad de relaciones casuales-designativas, sino, además, la formulación de tales relaciones a través de dos enunciados oracionales sinónimos desde el punto de vista de la significación oracional); de otro, relaciones entre enunciados que comparten sólo una representanción semejante desde el punto de vista designativo, que resulta a posteriori diversificada merced a su plasmación en esquemas sintácticos diferentes (como (6) y (7)) que, por lo tanto, no han de ser puestos en relación directa en este plano, sino en el más general de la designación.

6.1. En consecuencia, la organización del componente sintáctico de la gramática bajo esta nueva perspectiva sitúa en unos términos más correctos que los estándar-chomskyanos el tratamiento de oraciones activas y pasivas, que, al ser caracterizadas como esquemas oracionales diferentes desde un punto de vista estrictamente sintáctico-funcional, hacen impracticable la posibilidad de generación de las unas, las pasivas, como resultado de un proceso en el que necesariamente habrian de intervenir las otras, las activas.

7. La Semántica Generativa, especialmente en esta orientación fillmoriana, aun siendo - por las razones que acabamos de indicar- un estadio de enorme importancia en la evolución y conformación de la GGT como modelo coherente de descripción lingüística, no supone tampoco, sin embargo, el logro definitivo de una metodología de descripción gramatical plenamente satisfactoria. Continuando siempre en el marco de los problemas de los que es lícito esperar una clarificación en relación con las construcciones de tipo pasivo, la propuesta fillmoriana despierta, por su parte, algunas objeciones muy semejantes, en último extremo, a las formulables a modelos de corte más estrictamente chomskyano. Así, si bien, en el sentido de su valoración positiva, la adopción de la perspectiva casual permite una simplificación de la gramática haciendo, por ejemplo, innecesaria la ordenación cíclica de determinadas transformaciones como la de "cambio de lugar del complemento indirecto" y de pasiva que, como vimos, habían de operar en ese orden para poderse generar, en determinadas lenguas, aquellas oraciones pasivas cuyo sujeto corresponde al objeto indirecto de la oración activa; ordenación innecesaria en el marco de una propuesta como la de la Gramática de Casos, toda vez que Dativo y Objetivo 
serían, en tales lenguas, inmediata e igualmente seleccionables para la función de sujeto sin mayores restricciones, no es menos cierto que un modelo así elaborado sigue suscitando problemas importantes.

7.1. En primer lugar, y con referencia a la formulación de las reglas de conexión entre el nivel casual y el estrictamente sintáctico-funcional, las indicaciones fillmorianas sobre las condiciones que motivarían la selección de casos como Objetivo o Dativo para la función de sujeto, aunque aparentemente válidas, al descansar sobre un argumento explícito relativo a la estructura de la cadena oracional: la aparición del rasgo [+ pasiva] asociado a su Verbo, obedecen, a poco que se analicen detenidamente, a un razonamiento circular poco satisfactorio, al querer subordinar la aplicación de una transformación a la presencia en el núcleo predicativo de un rasgo que, básicamente, es de naturaleza morfológica y supone únicamente la aparición de un elemento auxiliar copulativo; caracterización que requeriria, parlalelamente, un estudio de las mutaciones de tipo funcional subyacentes a tal cambio morfológico en el predicado; y esto no sólo como requisito indispensable para una verdaderamente coherente descripción de los procesos funcionales envueltos en estructuras de este tipo, sino también como medio de operar una neta distinción respecto de las estructuras atributivas o copulativas, oraciones con las que las pasivas vendrían a coincidir inmediatamente, al menos por lo que a la estructura de sus predicados se refiere (16).

7.2. Paralelamente, la adopción del componente casual-predicativo como estadio básico dentro del nivel sintáctico de la gramática no arroja tampoco una claridad mayor en comparación con modelos de corte chomskyano-estándar sobre fenómenos como el de la imposibilidad de pasivización de estructuras con Agente y Objetivo o Dativo coreferenciales, para las que la posibilidad de selección como sujeto de elementos casuales distintos del Agente debería ser bloqueada por razones tan opacas como las que determinaban en el modelo choms-

(16) Cf. S. GILI GAYA, Curso Superior de Sintaxis española, Barcelona, Vox, 1979, pág. 122, y E. ALARCOS LLORACH, «La diátesis en español y «Pasividad y atribución en español”, en Estudios de gramática funcional del español, Madrid, Gredos, 1970, págs. 90-94 y 124132, respectivamente. 
kyano la necesidad última del concreto expediente de ordenación de las transformaciones de reflexivización y pasivización.

7.3. Finalmente, el tratamiento de la pasividad en un modelo como el de la Gramática de Casos parece poder ser dificilmente armonizado con el resultado de los estudios psicolingü ísticos mencionados en 3 . y que, si bien puedan ser discutibles en el sentido de su apoyo a la condición transformacional de las estructuras pasivas frente a la "básica» de las activas, no es menos cierto que dejan planteado el problema de la existencia de una conexión entre oraciones activas y pasivas que ha de resolverse, de alguna forma, poniendo de relieve una mayor complejidad de las estructuras pasivas por lo que a los procesos que en ellas son pertinentes se refiere.

7.3.1. Observaciones allegables por su intencionalidad última a éstas de cuño generativista son ciertamente encontrables entre gramáticos de orientaciones muy diferentes. Gili Gaya notaba ya, recordando de paso una actitud similar por parte de R.J. Cuervo, la tendencia de nuestra lengua hacia el uso, en situaciones gramaticales en que activas y pasivas serían igualmente posibles, de las activas; indicando que «el carácter nominal, estático, de las constru cciones pasivas con ser (asimilables a las atributivas), choca con la tendencia idiomática a preferir la construcción verbal, dinámica y animada, que se manifiesta también en otros puntos de nuestra Sintaxis» (17); Harald Weinrich, por su parte, habría cuantificado en torno a un $10 \%$ la manifestación o frecuencia, en textos orales alemanes, de las que denomina pasivas "completas"; es decir, aquéllas con expresión del agente, concluyendo que «la escasa frecuencia de esa estructura en textos reales (...) no justifica esta pasiva artificial, construida por los gramáticos para presentar el paralelismo de la diátesis" (18).

7.3.2. En cierta forma, la solución al problema de la descripción de las

(17) Cf. S. GILI GAYA, op. cit., pág. 22.

(18) Cf. H. WEINRICH, Lenguaje en textos, Madrid, Gredos, 1976, pág. 63 nota 6. 
estructuras oracionales pasivas por parte de Charles J. Fillmore puede, y debe, ser conectada con las actitudes precedentes, en la medida en que, expresamente, tales construcciones son consideradas como «menos normales" que las correspondientes activas, y se habla de la elección de Objetivo o Dativo como sujetos gramaticales como una «nonnormal choice" (19) frente a la "normal» del Agente. Sin embargo, una observación semejante, verdaderamente fundamental a propósido del tratamiento gramatical de estas construcciones, quedará sin ulteriores consecuencias desde el punto de vista de su reflejo en los concretos procedimientos meta-teóricos a través de los cuales han de ser tratadas en la gramática ya que, para poder ser coherentemente integrada dentro del modelo casual, éste debería poder distinguir explícitamente entre realizaciones normales y menos normales o no-normales, dentro de una misma esfera de gramaticalidad/aceptabilidad absolutas, para lo que no existe en este modelo, ni en ninguno de los modelos de GGT continuación de los planteamientos chomskyanos "estándar", recurso formal alguno.

8. La GGT y la Gramática de casos tienen en común en este punto su desarrollo como modelos concebidos sobre la base de una teoría freguiana de la categorización, según la cual, dado un elemento determinado, éste pertenece a una concreta categoría, $A$, o no lo hace, siendo, por tanto, necesariamente asignado a otra categoría diferente, $B$, según posea la totalidad de los rasgos definitorios que delimitan A y B como categorías (20). Según esto, siendo las oraciones pasivas esquemas estructurales en los que se produce una manifestación sintácticofuncional de determinadas relaciones casuales que no es posible reconducir o identificar con la que tiene lugar en las activas, deberá establecerse que ambos esquemas oracionales son el resultado de la aplicación de reglas diferentes que gobiernan el comportamiento de categorias oracionales también diferentes. Por la misma razón, perteneciendo ambos tipos de esquemas oracionales a estructuras categorialmente diferentes, resulta también imposible justificar el carácter "menos normal» de uno de ellos, por cuanto ello implicaría la necesidad de distinguir entre categorías de distinto grado de "normalidad», lo

(19) Ct. Ch. J. FILLMORE, op. cit., pág. 62.

(20) Para una visión crítica de la concepción freguiana, o « digital», de la categorización, Cf. E. ROSCH, aHuman Categorizationm, en Studies in Cross-cultural Psychology, vol l, ed. por Neil Warren, Nueva York, Academic Press, 1977, págs. 19 y ss. 
que, por definición, es un contrasentido, en la medida en que permitan albergar sin inconvenientes a aquellos elementos para cuya representación han sido establecidas.

8.1. Pero ni la condición de "no-normales» de las oraciones pasivas es imposible de asumir explícitamente dentro de un modelo formal, ni fenómenos como éstos dejan de tener ya una amplia tradición - si bien es cierto que no en el nivel sintáctico, sino más bien, en el morfológico $y$, especialmente, en el fono-fonológico- en modelos como los estructuralistas, ámbito metodológico que, lejos de constituir un aspecto de la historia de la lingüística carente de mayor interés - como injustamente fuera caracterizado por Noam Chomsky - contaba, al contrario, para la definición de los diferentes elementos gramaticales, junto a su categorización en distintas clases paradigmáticas, a través de las cuales era puesto de manifiesto el valor diferencial de cada elemento, con un recurso como el de la Neutralización, capaz de explicar de manera sistemática usos extensivos de valores sistemáticos, posibles en determinadas situaciones contextuales; y ello sin ser necesaria la ampliación del reticulado de clases paradigmáticas.

8.2. Por otra parte, un recurso como el de la neutralización ha encontrado, paradójicamente si se piensa en el juicio crítico negativo que la metodología estructural mereciera de la GGT, en los recientes estudios de Psicología cognitiva una confirmación que parece incuestionable, al mostrarse, a través de la consideración de ámbitos objetuales diferentes como el del color, las figuras geométricas o las expresiones faciales (21) cómo en la categorización de estos diferentes ámbitos se procede, no asignando necesariamente una categoría específica a cada situación objetual, sino, al contrario, elaborando categorías para acoger, básicamente, a determinados miembros que parecen representar ejemplarmente la categoría, y haciendo servir esas mismas categorías para albergar a otros elementos sólo parcialmente recubiertos por ellas, en la medida en que tales elementos se alejen de los elementos prototipicos, es decir, no compartan todas las propiedades de aquéllos sobre

(21) Una sintesis de tales investigaciones se encontrará en $\mathrm{E}$. ROSCH, *On the internal structure of perceptual and semantic categories $\infty$, en Cognitive Development and the acquisition of language, Nueva York, Academic Press, 1973, págs. 111-144. 
cuya imagen se ha elaborado la categoría que, por lo mismo, sólo corresponderá parcialmente (22) a tales elementos marginales.

9. Es en el ámbito de los fenómenos de neutralización o correspondencia parcial, que, me parece, pueden ser considerados semejantes, salvadas las diferencias de su uso en metodologías de carácter taxonómico y generativo, respectivamente, donde, en mi opinión, debe plantearse realmente el problema de las estructuras oracionales llamadas pasivas en lenguas como la nuestra, que no heredaron el sistema de diátesis verbal latino, $y$ en las que debió arbitrarse alguna solución para garantizar la posibilidad de transmisión del tipo de contenidos oracionales en que es ya tópicamente hecha consistir la llamada oración pasiva.

9.1. A pesar de que las gramáticas del español -al igual que las de otras muchas lenguas-, posiblemente por la asunción acrítica de categorías y problemas de las gramáticas clásicas, hayan mantenido el empeño por reservar dentro de la estructura de la Gramática un reticulado categorial injustificable para albergar una serie de fenómenos oracionales que se sentian como característicos, como ya se mostrara desde una perspectiva diferente (23), ni desde un punto de vista morfológico, ni desde una perspectiva morfo-sintáctica, es posible mantener la existencia en castellano de este tipo particular de construcciones.

9.2. Otra cuestión diferente es, sin embargo, la de la posibilidad de expresión de los contenidos sintáctico-funcionales denominados "pasivos"; problema gramatical que, como indicaba, puede ser resuelto merced a la introducción en el seno de un modelo generativo como aquél a que venimos haciendo referencia, de una teoría de la correspondencia parcial. Provistos de semejante recurso, en nada objetable, como hemos visto, por lo que a sus fundamentos psicológicos se re-

(22) Para algunas aplicaciones a problemas lingüisticos de semejante recurso, Cf. el ya citado trabajo de G. LAKOFF, “Linguistic Gestals», de alguna de cuyas soluciones descriptivas, especialmente en relación con la pasividad, disiento.

(23) Véase especialmente el par de estudios ya citados de E. ALARCOS. 
fiere, y recurriendo de nuevo al marco metodológico elaborado por Ch. $\mathrm{J}$. Fillmore, puede establecerse que, dada una estructura casualpredicativa en la que está presente un caso "Agente", como en (8), será éste seleccionado para la función de sujeto gramatical, como en (6); constituyendo semejante principio la regla en virtud de la cual las estructuras casual-predicativas son transformadas en esquemas sintáctico-funcionales como los tradicionalmente denominados activos; es decir, esquemas oracionales cuyo significado podría ser parafraseado, en términos aproximados, como indicando «la realización por un actor responsable de una acción que afecta a un destinatario».

9.2.1. Naturalmente, es posible imaginar buen número dé situaciones co/contextuales (24)en las que las implicaciones pragmáticas que de una estructura sintáctico-oracional tal se desprenden pueden ser no aconsejables, deseadas, etc. Así, correspondiendo, en términos generales, desde un punto de vista discursivo, la posición de sujeto a la categoría de Tópico (25), razones de naturaleza cotextual pueden hacer preferible/necesario asignar la condición de Tópico discursivo a un elemento casual distinto del Agente; por otra parte, conllevando la realización de una estructura casual-predicativa como (8) bajo la forma de un esquema oracional activo la indicación de la concreta identidad del actor responsable de la acción denotada por el predicado, o, al menos, en los casos de impersonalidad, la de "acción", resultaría también manifiesta la inadecuación de una estructura sintáctica semejante en situaciones contextuales en las que el locutor desea mantener un relativo distanciamiento con relación a los contenidos proposicionales de su enunciado (26).

9.2.2. En estos casos, la utilización de la construcción sintáctico-fun-

(24) Para la distinción cotexto (contexto lingüistico)/contexto (contexto extralingüistico), Cf. JANOS S. PETOFI, Vers une théorie partielle du texte, Papiere zur Textlinguistik, 9, 1975, pág. 1; y T. A. VAN DIJK, Some Aspects of Text Grammars, La Haya, Mouton, 1979, pág. 39.

(25) Cf. al respecto M. A. K. HALLIDAY, aEstructura y función del lenguaje", en Nuevos horizontes de la lingülstica, Madrid, Alianza Univ., 1970, págs. 145-174.

(26) La condición de adistancing device» de la pasividad ha sido destacada por ROBIN LAKOFF, "Linguistic theory and the real world", en Berkeley Studies in Syntax and Semantics, Berkeley, California, 1974, vol. I, pág. 9. 
cional paradigmáticamente correspondiente a estructuras casual-predicativas en las que está presente un Agente resultaría inadecuada de acuerdo con exigencias comunicativas puntuales como las apuntadas; pero puede ser evitada si, en lugar de hacer corresponder tales contenidos con el esquema sintáctico predicativo tradicionalmente denominado "activo" (con el que paradigmática o prototípicamente se corresponden), esos mismos contenidos son incardinados en un tipo estructural oracional diferente, pero parcialmente similar: el atributivo, dentro del cual el elemento casual jerárquicamente dominante para la posición de sujeto oracional es el Objetivo o Dativo; proceso que puede ser extrapolado para el caso de estructuras como (8), siempre que se cumpla la condición de acomodar, paralelamente, la estructura del elemento predicativo nuclear a la que es característica de los esquemas sintácticos atributivos; es decir, se confiera al Predicado de (8) una estructura en la que esté presente un elemento copulativo más un núcleo semántico que es paradigmáticamente adjetivo (lo que, en cierta forma, explicaría la estructura verbal pasiva, integrada por un elemento copulativo y una forma de participio); convirtiendo así una determinada representación casual-predicativa en un esquema sintáctico-funcional cuyo significado no es, como lo sería en el caso de ser transmitido a través de un esquema como el predicativo/activo, el de «actor que ejecuta una acción sobre un destinatario", sino el de "Destinatario en una determinada situación (originada por/debida a un agente)»; 0 , si se desea, neutralizando la diferencia entre proposiciones, en términos greimasianos, funcionales y cualificativas (27), y haciendo que las últimas sirvan como archiforma de la oposición neutralizada, lo que, de otra parte, explicaría la doble interpretación de (7) como:

i) atributiva; en lo que conlleva de indicación de una cualificación referida a un actante-Objetivo.

ii) predicativa, en su componente de acción, y en la indicación del agente responsable.

10. Una descripción semejante encierra considerables ventajas respecto a los tratamientos de la pasivilidad previamente reseñados en los modelos generativos. En primer lugar, al mismo tiempo que se considera a oraciones activas y pasivas como esquemas oracionales en estricta relación parafrástica respecto de unos mismos

(27) Vid. nota (9). 
contenidos casual-predicativos, permite dar cuenta de lo anómato o especial de la solución pasiva, resultado de su condición de esquema producto de la utilización en «correspondencia parcial» de la estructura oracional atributiva. Por otra parte, el recurso a esta correspondencia parcial podría ser considerado como sustentando las conclusiones de los estudios psicolingüísticos aludidos, permitiendo su interpretación en un sentido diferente, y no necesariamente probatorio de la existencia de la transformación pasiva, toda vez que la mayor dificultad en el procesar las construcciones pasivas, el mayor tiempo requerido para ello, etc., etc., puede perfectamente ser considerado como resultado de la condición no prototípica o paradigmática de estas construcciones respecto de las estructuras atributivas.

10.1. Un tratamiento de la pasividad como el propuesto aporta, de otra parte, una inmediata justificación para algunos de los, de otra forma, opacos procedimientos transformacionales en relación con las oraciones pasivas. En concreto, la imposibilidad de pasivización de estructuras con Agente y Objetivo/Dativo co-referenciales resultaría ser absolutamente lógica a partir de una explicación de las oraciones pasivas como la que propongo, ya que carecería de sentido el intento de evitar la condición de sujeto gramatical para el Agente, cuando la alternativa es su sustitución por otro elemento referencialmente idéntico a él.

11. La asunción de procedimientos como el indicado en el seno de un modelo generativo conlleva, sin embargo, inevitablemente, modificaciones sustanciales de algunos de los presupuestos fundamentales sobre los que las GGGGTT han sido establecidas.

11.1 En primer lugar, obliga necesariamente a desechar la condición impuesta por Noam Chomsky en el sentido de que las reglas de una gramática generativa han de ser aplicables de manera automática, y en virtud sólo del conocimiento de los elementos puntualmente existentes en un estadio determinado del proceso de derivación, para postular, al contrario, que, al menos en determinados casos, la actuación de una regla o proceso generativo implica necesariamente el conocimiento de sus implicaciones co/contextuales, asi como el de las de otros esque- 
mas alternativos (28); lo que, de alguna manera parece apuntar a la necesidad de reinstauración, en el ámbito de los modelos generativos, de una imagen de la competencia como conjunto de clases de reglas "conocidas" opositivamente por los hablantes.

11.2. En segundo lugar, la asunción de un mecanismo como el de correspondencia parcial obliga también a cuestionar los términos en los que fuera establecida por Chomsky la oposición entre los conceptos de competencia y actuación, en el sentido de la no pertenencia al ámbito de intereses estrictamente lingüísticos salvo de aquellas cuestiones relativas a la competencia. Bien al contrario, tan sólo en la medida en que los fenómenos de actuación sean tenidos en cuenta, mecanismos como los de correspondencia parcial, o neutralización, podrán ser coherentemente incorporados a la gramática atendiendo al pleno sentido de su funcionalidad. Paradójicamente, por tanto, tan sólo en la medida en que la actuación no sea dejada al margen de los intereses lingüísticogramaticales, será posible acceder a una verdadera comprensión de los mecanismos de competencia; nivel que, desconectado del de la actuación, cuando no resulta caracterizado poco satisfactoriamente, como he intentado mostrar a propósito del caso concreto de las estructuras oracionales pasivas, correrá siempre el riesgo de ser esquematizado pobremente por la falta de toma en consideración, junto a los valores funcionales centrales o paradigmáticos de las diferentes categorias, de sus valores menos centrales o parciales.

(28) Con el nombre de "transderivational rules», G. LAKOFF ha apuntado a fenómenos semejantes; cf. G. LAKOFF, "Some thoughts on transderivational rules", en Papers in Linguistcis in honor of H. and R. Kahane, ed. por B. Kachru y alt., Univ. of Illinois Press, 1973. 